\title{
Nonlinear Estimation for Position-Aided Inertial Navigation Systems
}

\author{
Soulaimane Berkane and Abdelhamid Tayebi
}

\begin{abstract}
In this work we solve the position-aided 3D navigation problem using a nonlinear estimation scheme. More precisely, we propose a nonlinear observer to estimate the full state of the vehicle (position, velocity, orientation and gyro bias) from IMU and position measurements. The proposed observer does not introduce additional auxiliary states and is shown to guarantee semi-global exponential stability without any assumption on the acceleration of the vehicle. The performance of the observer is shown, through simulation, to overcome the state-of-the-art approach that assumes negligible accelerations.
\end{abstract}

\section{INTRODUCTION}

Inertial navigation systems (INS) are essential devices that allow the localization and control of autonomous vehicles and robot platforms [1]. Classical INS fuse measurements from onboard accelerometers and gyroscopes (typically included in an Inertial Measurement Unit (IMU)), to continuously compute the position, velocity and orientation of a vehicle without any external reference measurement. However, this classical approach (known also as dead reckoning) suffers from drift due to measurement errors and unknown initial conditions [2]. As a result, inertial navigation systems are often assisted by position sensors such as the Global Positioning System (GPS), which allow for the correction of position estimates over time, resulting in small and bounded estimation errors, see, e.g., [3], [4], [5]. Other type of sensors that can provide range (distance) measurements to known source points can also be used to provide position information such as GPS pseudo-ranges [6], [7], [8] or Ultra Wide-Band (UWB) radio technology [9], [10]. Kalman-type filters, such as [11], [4], [12], [13], are considered industry-standard solutions for inertial navigation systems. However, these filters are often based on linearization assumptions and may fail when the initial estimation errors are large. On the other hand, nonlinear observers have been developed for autonomous navigation applications; see for instance [7], [14], [8], [15], [16], [17], [18]. The advantage of the nonlinear observers is their theoretically proven stability guarantees, as well as their computational simplicity compared to the stochastic filters.

In this work, we propose a nonlinear observer for the simultaneous estimation of the position, linear velocity, attitude, and gyro bias of a rigid body system. The estimator relies

This work was supported by the National Sciences and Engineering Research Council of Canada (NSERC), under the grants NSERCDG RGPIN-2020-04759 and NSERC-DG RGPIN-2020-0627. S. Berkane (soulaimane.berkane@uqo.ca) is with the Department of Computer Science and Engineering, University of Quebec in Outaouais, Gatineau, QC, Canada. A. Tayebi (atayebi@lakeheadu.ca) is with Department of Electrical Engineering, Lakehead University, Thunder Bay, ON, Canada. on IMU and inertial position measurements which are used in typical navigation scenarios. The attitude estimates are directly obtained on the Special Orthogonal group of rotations $\mathbb{S O}(3)$, thus avoiding any singularities or ambiguities related to the use of other attitude parameterizations. The proposed observer, evolving on $\mathbb{S O}(3) \times \mathbb{R}^{9}$ is shown to guarantee semi-global exponential stability, which is the strongest stability result that can be achieved on this state space using smooth observers. In contrast to [5], [7], [15], [18], the proposed observer does not require the introduction of an auxiliary 3-dimensional state in the estimation scheme, thus reducing the computational burden associated with the real-time implementation of the estimator. The paper is structured as follows. Preliminaries are provided in Section [I] while the problem at hand is formulated in Section III The observer design and the corresponding stability result are provided in Section IV (main result). Simulation results using an accelerated trajectory are given in Section $\mathrm{V}$ to show the performance of the proposed observer and to compare it with a practical adhoc estimation scheme. We conclude the paper with some remarks in Section VI

\section{BACKGROUND}

We denote by $\mathbb{R}$ the set of reals and by $\mathbb{N}$ the set of natural numbers. We denote by $\mathbb{R}^{n}$ the $n$-dimensional Euclidean space, by $\mathbb{S}^{n}$ the unit $n$-sphere embedded in $\mathbb{R}^{n+1}$ and by $\mathbb{B}_{\epsilon}=\left\{x \in \mathbb{R}^{3}:\|x\| \leq \epsilon\right\}$ the closed ball in $\mathbb{R}^{3}$ with radius $\epsilon$. We use $\|x\|$ to denote the Euclidean norm of a vector $x \in \mathbb{R}^{n}$ and $\|A\|_{F}$ to denote the Frobenius norm of a matrix $A \in \mathbb{R}^{n \times n}$. Let $I_{n}$ be the $n$-by- $n$ identity matrix and let $e_{i}$ denote the $i$-th column of $I_{n}$. The Special Orthogonal group of order three is denoted by $\mathbb{S O}(3):=$ $\left\{A \in \mathbb{R}^{3 \times 3}: \operatorname{det}(A)=1, A A^{\top}=A^{\top} A=I_{3}\right\}$. The set $\mathfrak{s o}(3):=\left\{\Omega \in \mathbb{R}^{3 \times 3} \mid \Omega^{\top}=-\Omega\right\}$ denotes the Lie algebra of $\mathbb{S O}(3)$. For $x, y \in \mathbb{R}^{3}$, the map $[\cdot]_{\times}: \mathbb{R}^{3} \rightarrow \mathfrak{s o}(3)$ is defined such that $[x]_{\times} y=x \times y$ where $\times$ is the vector cross-product on $\mathbb{R}^{3}$. The inverse isomorphism of the map $[\cdot]_{\times}$is defined by vex $: \mathfrak{s o}(3) \rightarrow \mathbb{R}^{3}$, such that $\operatorname{vex}\left([\omega]_{\times}\right)=\omega$, for all $\omega \in \mathbb{R}^{3}$ and $[\operatorname{vex}(\Omega)]_{\times}=\Omega$, for all $\Omega \in \mathfrak{s o}(3)$. The composition map $\psi:=\operatorname{vex} \circ \mathbf{P}_{\mathfrak{s o}(3)}$ extends the definition of vex to $\mathbb{R}^{3 \times 3}$, where $\mathbf{P}_{\mathfrak{s o}(3)}: \mathbb{R}^{3 \times 3} \rightarrow \mathfrak{s o}(3)$ is the projection map on the Lie algebra $\mathfrak{s o}(3)$ such that $\mathbf{P}_{\mathfrak{s o}(3)}(A):=\left(A-A^{\top}\right) / 2$. Accordingly, for a given 3-by-3 matrix $A=\left[a_{i j}\right]_{i, j=1,2,3}$, one has $\psi(A)=\frac{1}{2}\left[a_{32}-a_{23}, a_{13}-a_{31}, a_{21}-a_{12}\right]$. We define $|R|:=\frac{1}{4} \operatorname{tr}\left(I_{3}-R\right)=\frac{1}{8}\left\|I_{3}-R\right\|_{F}^{2} \in[0,1]$ as the normalized Euclidean distance on $\mathbb{S O}(3)$. Given a scalar $c>0$, we define the saturation function sat ${ }_{c}: \mathbb{R}^{n} \rightarrow \mathbb{R}^{n}$ such that $\operatorname{sat}_{c}(x):=\min (1, c /\|x\|) x$. Given two scalars $c, \epsilon>0$, we 
also define the smooth projection function $\mathbf{P}_{c}^{\epsilon}: \mathbb{R}^{3} \times \mathbb{R}^{3} \rightarrow \mathbb{R}^{3}$, found for instance in [19], as follows:

$$
\mathbf{P}_{c}^{\epsilon}(\hat{\phi}, \mu):= \begin{cases}\mu, & \text { if }\|\hat{\phi}\|<c \text { or } \hat{\phi}^{\top} \mu \leq 0, \\ \left(I-\theta(\hat{\phi}) \frac{\hat{\phi} \hat{\phi}^{\top}}{\|\|^{2}}\right) \mu, & \text { otherwise, }\end{cases}
$$

where $\theta(\hat{\phi}):=\min (1,(\|\hat{\phi}\|-c) / \epsilon)$. The projection operator $\mathbf{P}_{c}^{\epsilon}(\hat{\phi}, \mu)$ is locally Lipschitz in its arguments. Moreover, provided that $\|\phi\| \leq c$, the projection map $\mathbf{P}_{c}^{\epsilon}(\hat{\phi}, \mu)$ satisfies, along the trajectories of $\dot{\hat{\phi}}=\mathbf{P}_{c}^{\epsilon}(\hat{\phi}, \mu),\|\hat{\phi}(t)\| \leq c+\epsilon, \forall t \geq 0$.

\section{Problem Formulation}

Consider the following dynamics of a rigid-body vehicle:

$$
\begin{aligned}
\dot{R} & =R[\omega]_{\times}, \\
\dot{p} & =v, \\
\dot{v} & =g e_{3}+R a_{B},
\end{aligned}
$$

where $p \in \mathbb{R}^{3}$ is the inertial position of the vehicle's center of gravity, $v \in \mathbb{R}^{3}$ represents the inertial linear velocity, $R \in$ $\mathbb{S O}(3)$ is the rotation matrix describing the orientation of the body-attached frame with respect to the inertial frame, $\omega$ is the angular velocity of the body-attached frame with respect to the inertial frame expressed in the body-attached frame, $g$ is the norm of the acceleration due to gravity, $e_{3}=[0,0,1]^{\top}$ and $a_{B}=R^{\top} a_{I}$ is the "apparent acceleration", capturing all non-gravitational forces applied to the vehicle, expressed in the body-attached frame.

We assume available an inertial measurement unit (IMU) that provides measurements in the body frame of the angular velocity, the apparent acceleration and the earth's magnetic field. These sensors are modelled as follows:

$$
\begin{aligned}
\omega^{y} & =\omega+b_{\omega}, \\
a_{B} & =R^{\top} a_{I}, \\
m_{B} & =R^{\top} m_{I},
\end{aligned}
$$

where $b_{\omega}$ is a constant unknown gyro bias, $m_{I}$ is the constant and know earth's magnetic field and $a_{I}(t)$ is a time-varying unknown apparent acceleration. For the rotational dynamics, the following is a general observability assumption commonly used in attitude estimation.

Assumption 1. There exists a constant $c_{0}>0$ such that $\| m_{I} \times$ $a_{I}(t) \| \geq c_{0}$ for all $t \geq 0$.

Assumption 1 is guaranteed if the time-varying apparent acceleration $a_{I}(t)$ is non-vanishing and is always non-collinear to the constant magnetic field vector $m_{I}$. Note that $a_{I}(t)=0$ corresponds to the rigid body in a free-fall $\left(\dot{v}=g e_{3}\right)$ which is not likely under normal flight conditions.

We also assume that we have measurements of the following position output vector:

$$
y=C_{p} p,
$$

where $C_{p} \in \mathbb{R}^{m \times 3}, m \in \mathbb{N}$, is a given output matrix that satisfies $\operatorname{rank}\left(C_{p}\right)=3$. Roughly speaking, this rank assumption means that the measurement $y$ is sufficient for the construction of a converging translational observer (assuming perfect knowledge of the attitude) for (3)-(4). The measurement $y$ can be obtained from different possible sensors, depending on the application at hand, that provide some information about the position. For full position measurements obtained from a GPS for instance, $C_{p}=I_{3}$. For range measurements, obtained from Ultra-Wide Brand (UWB) sensors for instance, the output $y$ and the matrix $C_{p}$ can be obtained from the range measurements as done in [18, Section 4.3.2]; see also the simulation work in Section $\mathrm{V}$

The objective of this work is to design a full navigation system that processes the measurements (7)-(8) and outputs reliable estimates for the position $p \in \mathbb{R}^{3}$, the linear velocity $v \in \mathbb{R}^{3}$, the orientation $R \in \mathbb{S O}(3)$, and the gyro bias $b_{\omega} \in$ $\mathbb{R}^{3}$. More specifically, our goal is to design an exponentially convergent nonlinear observer that estimates the whole state of the vehicle using an observer state that evolves on the same manifold as the system state $\left(\mathbb{S O}(3) \times \mathbb{R}^{9}\right)$. We further consider the following mild (realistic) constraints on the trajectory of the vehicle which are needed to prove the main result:

Assumption 2. There exist constants $c_{1}, c_{2}, c_{3}>0$ such that $c_{1} \leq\left\|a_{I}(t)\right\| \leq c_{2}$ and $\left\|\dot{a}_{I}(t)\right\| \leq c_{3}$ for all $t \geq 0$.

Assumption 3. There exists constants $c_{4}, c_{5}>0$ such that $\|\omega(t)\| \leq c_{4}$ and $\left\|b_{\omega}\right\| \leq c_{5}$ for all $t \geq 0$.

\section{Observer Design and Stability Analysis}

In this section, we design a navigation observer to estimate the full state $\left(p, v, R, b_{\omega}\right)$ consisting of the position, velocity, orientation and gyro bias. We define the combined translational state $x:=\left[p^{\top}, v^{\top}\right]^{\top} \in \mathbb{R}^{6}$. Then, in view of (3)-(4) and (8), the dynamics of $x$ are written as:

$$
\begin{aligned}
& \dot{x}=A x+B\left(g e_{3}+R a_{B}\right), \\
& y=C x,
\end{aligned}
$$

where the matrices $A, B$ and $C$ are defined as follows:

$$
A=\left[\begin{array}{cc}
0_{3 \times 3} & I_{3} \\
0_{3 \times 3} & 0_{3 \times 3}
\end{array}\right], B:=\left[\begin{array}{c}
0_{3 \times 3} \\
I_{3}
\end{array}\right], C=\left[\begin{array}{c}
C_{p}^{\top} \\
0_{3 \times m}
\end{array}\right]^{\top} .
$$

The translational dynamics $90-(10)$ are those of a linear timeinvariant system with unknown input $a_{I}=R a_{B}$ representing the apparent acceleration which is known only in the bodyframe. A common approach, in practice, consists in assuming that the acceleration of the vehicle is negligible, i.e., $\dot{v} \approx 0$, and therefore $a_{I} \approx-g e_{3}$. Under this small linear acceleration assumption, the attitude and gyro bias estimation can be done separately using the following explicit complementary filter proposed in [20], [5]:

$$
\begin{aligned}
\dot{\hat{R}} & =\hat{R}\left[\omega_{y}-\hat{b}_{\omega}+k_{R} \sigma_{R}\right]_{\times}, \\
\dot{\hat{b}}_{\omega} & =\mathbf{P}_{c_{5}}^{\epsilon_{b}}\left(\hat{b}_{\omega},-k_{b} \sigma_{R}\right),
\end{aligned}
$$

with the innovation term

$$
\sigma_{R}=\rho_{1}\left(m_{B} \times \hat{R}^{\top} m_{I}\right)+\rho_{2}\left(a_{B} \times \hat{R}^{\top}\left(-g e_{3}\right)\right) .
$$

Once the attitude is estimated, the translational motion state $x$ can be estimated using a Luenberger-like observer as follows:

$$
\dot{\hat{x}}=A \hat{x}+B\left(g e_{3}+\hat{R} a_{B}\right)+K(y-C \hat{x}),
$$




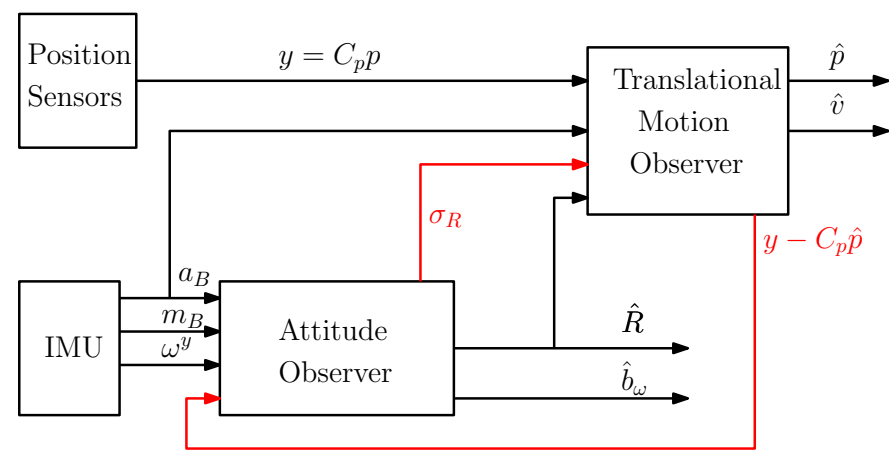

Fig. 1. Overall structure of the proposed nonlinear observer in 16$]-(18)$. The observer uses an IMU and position information to estimate the whole state of the vehicle. Compared to the adhoc estimation scheme $(12)-(15)$, the proposed observer introduces coupling (in bold red) between the translational estimator and the rotational estimator through their innovation terms. This additional coupling is important to guarantee the stability of the observer without the low acceleration assumption.

where $K$ is a gain matrix guaranteeing that $(A-K C)$ is Hurwitz, which can be either constant or tuned via a Riccati equation such as in the Kalman filter. The cascaded adhoc estimation scheme (12)-(15) will be used as a state-of-the-art algorithm in our comparison results in Section $\mathrm{V}$

In this work, however, we design our estimation algorithm without the small acceleration assumption which compromises the performance of the adhoc scheme when the vehicle is subject to large linear accelerations. In particular, we propose the following nonlinear navigation observer on $\mathbb{S O}(3) \times \mathbb{R}^{9}$ :

$$
\begin{aligned}
\dot{\hat{R}} & =\hat{R}\left[\omega_{y}-\hat{b}_{\omega}+k_{R} \sigma_{R}\right]_{\times}, \\
\dot{\hat{b}}_{\omega} & =\mathbf{P}_{c_{5}}^{\epsilon_{b}}\left(\hat{b}_{\omega},-k_{b} \sigma_{R}\right), \\
\dot{\hat{x}} & =A \hat{x}+B\left(g e_{3}+\hat{R} a_{B}\right)+K(y-C \hat{x})+\sigma_{x},
\end{aligned}
$$

with initial conditions $\hat{x}(0) \in \mathbb{R}^{6}, \hat{R}(0) \in \mathbb{S O}(3)$ and $\hat{b}_{\omega}(0) \in$ $\mathbb{B}\left(c_{5}+\epsilon_{b}\right)$. The innovation terms $\sigma_{x}:=\left[\sigma_{p}^{\top}, \sigma_{v}^{\top}\right]^{\top} \in \mathbb{R}^{6}$ and $\sigma_{R} \in \mathbb{R}^{3}$ are defined as follows:

$\sigma_{v}=K_{p} C_{p} \sigma_{p}$,

$\sigma_{p}=k_{R}\left(K_{v} C_{p}\right)^{-1}\left[\hat{R} \sigma_{R}\right]_{\times} \hat{R} a_{B}$,

$\sigma_{R}=\rho_{1}\left(m_{B} \times \hat{R}^{\top} m_{I}\right)+\rho_{2}\left(a_{B} \times \hat{R}^{\top} \operatorname{sat}_{\hat{c}_{2}}\left(K_{v}(y-C \hat{x})\right)\right)$.

The constant scalars $k_{R}, k_{b}, \rho_{1}, \rho_{2}, \epsilon_{b}, \hat{c}_{2}$ are positive with $\hat{c}_{2}>\sqrt{8} c_{2}$, the gain matrix $K=\left[\begin{array}{ll}K_{p}^{\top} & K_{v}^{\top}\end{array}\right]^{\top} \in \mathbb{R}^{6 \times m}$ is chosen as $K=L_{\gamma} K_{0}$ such that $A-K_{0} C$ is Hurwitz, $L_{\gamma}=\operatorname{blockdiag}\left(\gamma I_{3}, \gamma^{2} I_{3}\right)$ and $\gamma \geq 1$. Note that the inverse of the matrix $\left(K_{v} C_{p}\right)$ in 20 exists since $\left(A-K_{0} C\right)$ is a $2 \times 2$ invertible block matrix and the Schur complement of $I_{3}$ is the matrix $-K_{v} C_{p}$; see [21, Theorem 2.2]. The overall structure of the proposed estimation algorithm is depicted in Fig. 1. Compared to the adhoc estimation scheme (12)(15), the proposed observer introduces coupling through $\sigma_{x}$ and $\sigma_{R}$ between the translational estimator and the rotational estimator. This additional coupling is important to guarantee the stability of the observer without the low acceleration assumption. We define the following estimation errors:

$$
\begin{aligned}
\tilde{x} & :=x-\hat{x}, \\
\tilde{R} & :=R \hat{R}^{\top}, \\
\tilde{b}_{\omega} & :=b_{\omega}-\hat{b}_{\omega} .
\end{aligned}
$$

In view of (2)-(3) (17)-(18) and (22)-(24) one can derive the following dynamics for the estimation errors:

$$
\begin{aligned}
\dot{\tilde{x}} & =(A-K C) \tilde{x}+B(I-\tilde{R})^{\top} a_{I}-\sigma_{x}, \\
\dot{\tilde{R}} & =\tilde{R}\left[-\hat{R}\left(\tilde{b}_{\omega}+k_{R} \sigma_{R}\right)\right]_{\times}, \\
\dot{\tilde{b}}_{\omega} & =\mathbf{P}_{c_{5}}^{\epsilon_{b}}\left(\hat{b}_{\omega}, k_{b} \sigma_{R}\right) .
\end{aligned}
$$

To prove the convergence of the estimation errors, we introduce the following auxiliary error variable

$$
\zeta:=L_{\gamma}^{-1}\left[(A-K C) \tilde{x}+B(I-\tilde{R})^{\top} a_{I}\right] .
$$

The term between brackets in $(28)$ is an estimation error term that contains a suitable coupling between the translational estimation error $\tilde{x}$ and the rotational estimation error $\tilde{R}$. This coupling in the estimation errors, obtained from (25), allows to design the innovation term $\sigma_{x}$ by inspecting the dynamics of this coupled estimation error. This coupling is instrumental in showing (exponential) stability of the overall closed-loop system without requiring an additional observer state as done in our previous work [18]. Moreover, the matrix $L_{\gamma}^{-1}$ is introduced to assign a certain time-scaling structure between the different estimation errors in the same spirit as in [18]; see also [22], [23] for a motivation of this use in the context of high-gain observers. The high-gain $\gamma$ allows to deal efficiently with the interconnection between the rotational and translational dynamics. Now we are ready to state our main result.

Theorem 1. Consider the interconnection of the dynamics (3)(2) with the observer (18)-(21) where Assumptions (11)-(3) are satisfied. For each $\epsilon \in\left(\frac{1}{2}, 1\right)$ and for all initial conditions such that $\zeta(0) \in \mathbb{R}^{6}, \tilde{R}(0) \in \mho(\epsilon)=\{\tilde{R}:|\tilde{R}(0)| \leq \epsilon\}$ and $\hat{b}_{\omega}(0) \in \mathbb{B}\left(c_{5}+\epsilon_{b}\right)$, there exist $k_{R}^{*}>0$ and $\gamma^{*} \geq 1$ such that, for all $k_{R} \geq k_{R}^{*}$ and $\gamma \geq \gamma^{*}$, the estimation errors $\left(\zeta, \tilde{R}, \tilde{b}_{\omega}\right)$ are globally uniformly bounded and converge exponentially to zero.

\section{Proof. See Appendix A}

Theorem 1 shows that the proposed navigation observer (16)-21) guarantees exponential convergence of the estimation errors to zero starting from any initial condition inside the set $\mho(\epsilon)$. As $\epsilon$ tends to 1 , the set $\mho(\epsilon)$ covers the set of all attitude errors with rotation angle less than $180^{\circ}$. The derived bounds on the gains $k_{R}$ and $\gamma$ (see the proof) are expected to be very conservative as demonstrated in the simulation results.

\section{Simulation}

In this section, we simulate the proposed nonlinear estimator presented in Section IV] in the case of position range 
measurements. The real position trajectory of the vehicle is given by:

$$
p(t)=\left[\begin{array}{c}
\cos \left(2 \pi t^{2} / 100\right) \\
\sin \left(2 \pi t^{2} / 100\right) \\
1
\end{array}\right] .
$$

This corresponds to a circular trajectory with an angular frequency that increases over time (given by $t / 100$ ). The attitude trajectory is generated using the following angular velocity:

$$
\omega(t)=\left[\begin{array}{c}
\sin (0.2 t) \\
\cos (0.1 t) \\
\sin (0.3 t+\pi / 6)
\end{array}\right],
$$

and an initial attitude $R(0)=\exp \left(\left[\pi e_{1} / 2\right]_{\times}\right)$. The gyro measurements are corrupted by a constant bias of $3(\mathrm{deg} / \mathrm{sec})$ in each axis. The inertial earth's magnetic field is taken as $m_{I}=\left[\begin{array}{lll}0.033 & 0.1 & 0.49\end{array}\right]^{\top}$ and the earth's gravity is $g=9.81\left(\mathrm{~m} / \mathrm{sec}^{2}\right)$. We also assume available four noncoplanar source points for range sensing located at:

$$
\begin{aligned}
& a_{1}=\left[\begin{array}{lll}
1 & 1 & 2
\end{array}\right]^{\top}, \\
& a_{2}=\left[\begin{array}{lll}
1 & 3 & 0
\end{array}\right]^{\top} \text {, } \\
& a_{3}=\left[\begin{array}{lll}
0 & 1 & 1
\end{array}\right]^{\top} \text {, } \\
& a_{4}=\left[\begin{array}{lll}
6 & 5 & 5
\end{array}\right]^{\top} \text {. }
\end{aligned}
$$

The corresponding range measurements are given by

$$
d_{i}=\left\|p-a_{i}\right\|, \quad i=1, \cdots, 4 .
$$

To obtain an output equation of type (8) we proceed as follows. Define the following measurable scalars

$$
y_{i}=\frac{1}{2}\left(d_{i}^{2}-d_{1}^{2}-\left\|a_{i}\right\|^{2}+\left\|a_{1}\right\|^{2}\right), \quad i=2, \cdots, 4 .
$$

Then, one can show that $y_{i}=\left(a_{1}-a_{i}\right)^{\top} p, i=2, \cdots, 4$. Define $y=\left[y_{2}, \cdots, y_{4}\right]^{\top}$ one has

$$
y=\left[\begin{array}{c}
\left(a_{1}-a_{2}\right)^{\top} \\
\left(a_{1}-a_{3}\right)^{\top} \\
\left(a_{1}-a_{4}\right)^{\top}
\end{array}\right] p:=C_{p} p .
$$

It can be verified that $C_{p}$ has a rank of 3 .

The initial conditions for the observer states are $\hat{p}(0)=$ $\hat{v}(0)=\hat{b}_{\omega}(0)=0$ and $\hat{R}(0)=I_{3}$. The parameters of the observer are selected as $k_{R}=2, k_{b}=1, \rho_{1}=\rho_{2}=$ $1, \epsilon_{b}=0.001, \hat{c}_{2}=9 \sqrt{8}$ and $\gamma=2$. The gain $K_{0}$ is tuned such that the matrix $\left(A-K_{0} C\right)$ has eigenvalues at $\{-3,-3,-3,-4,-4,-4\}$. The adhoc estimator (12)-(15) is implemented with the same parameters and initial conditions. The simulation results given in Figures 2 -6 show that the proposed observer is able to estimate the position, velocity, acceleration, attitude and gyro bias using IMU and range measurements. On the other hand, as the acceleration of the vehicle increases, the adhoc estimator drifts away from the true trajectory while the proposed estimator is stable against high accelerations.

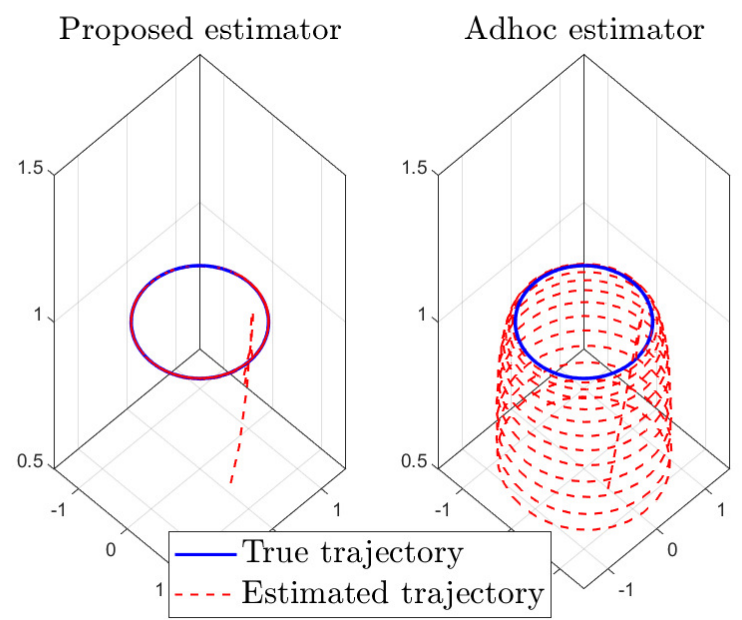

Fig. 2. True and estimated trajectory of the vehicle for the proposed observer which is compared against the adhoc estimator that assumes negligible accelerations. The video of the simulation can be found at https://youtu.be/ zbkSDZgh3vU
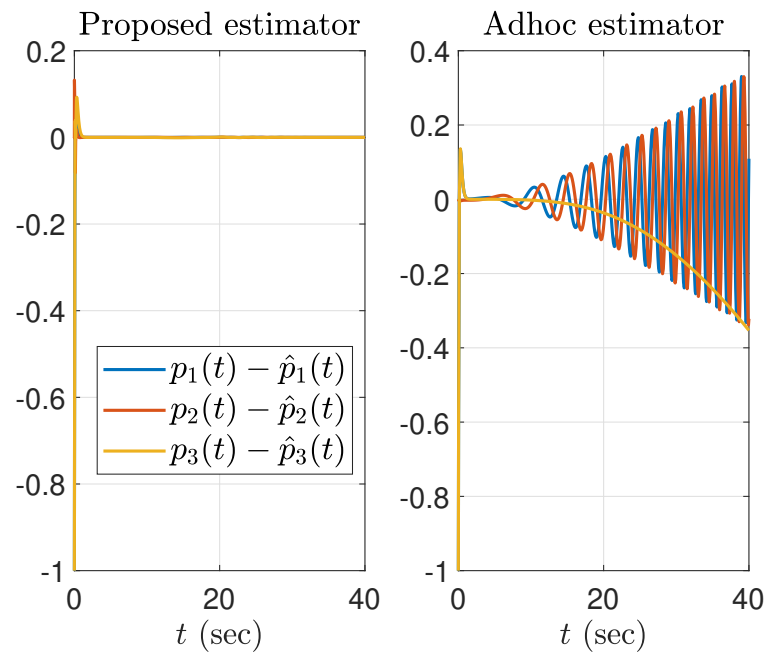

Fig. 3. Position estimation error.
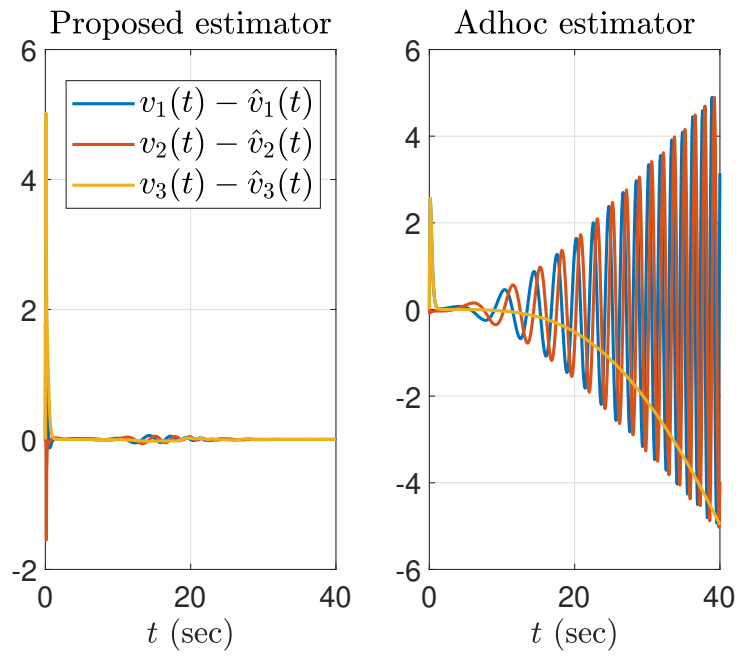

Fig. 4. Velocity estimation error. 

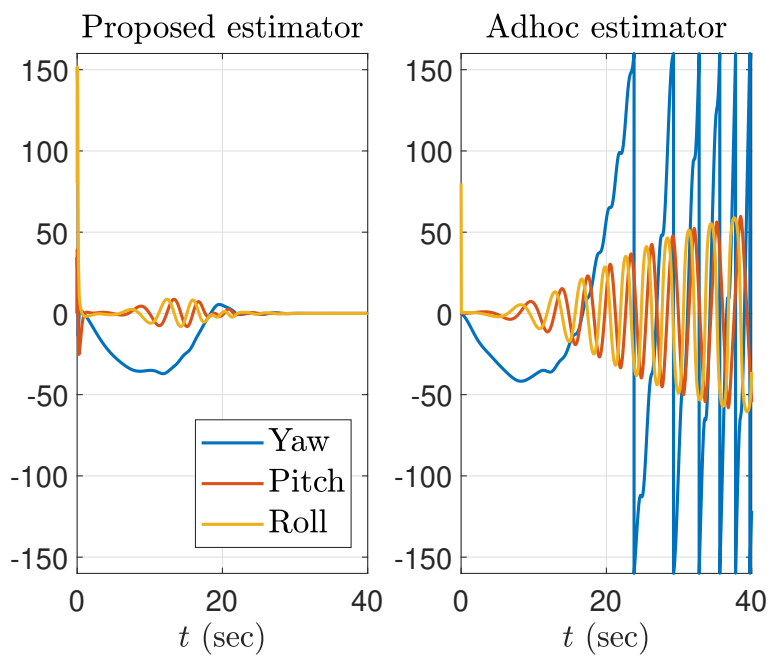

Fig. 5. Euler angles (Roll, Pitch, Yaw) corresponding to the attitude estimation error $\tilde{R}=R \hat{R}^{\top}$.
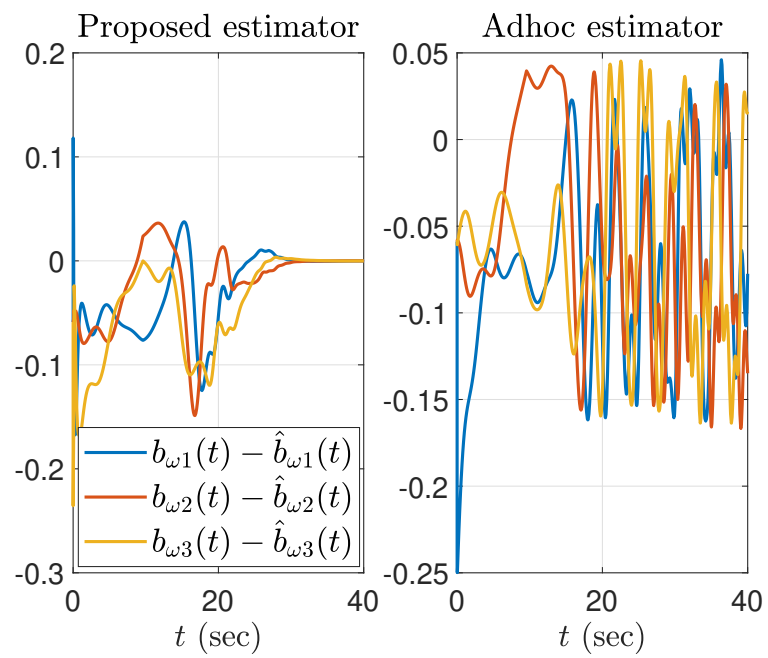

Fig. 6. Gyro bias estimation error.

\section{CONCLUSION}

In this paper we solved the full state (position, velocity, orientation, gyro bias) estimation problem for vehicles navigating in 3-dimensional spaces. The proposed solution is based on a nonlinear observer evolving on the configuration space $\mathbb{S O}(3) \times \mathbb{R}^{9}$ that uses IMU and position measurements as inputs and guarantees semi-global exponential stability. The proposed solution is particularly suitable in applications with important accelerations where the traditional cascaded approach (attitude estimation + position estimation) fails due to the small-accelerations assumption. The key feature of this observer is the fact that it does not add computational complexity compared to the traditional approach whereas our previous solution in [18] requires the introduction of auxiliary states to achieve the same stability result.

\section{APPENDIX A \\ Proof of THEOREM 1}

The proof of this result is mainly inspired from our proof in [18] with few modifications related to the particular closedloop dynamics at hand. First, we provide the following easyto-check facts:

$$
L_{\gamma}^{-1} A L_{\gamma}=\gamma A, \quad L_{\gamma}^{-1} B=\gamma^{-2} B, \quad C L_{\gamma}=\gamma C .
$$

Moreover, in view of the expression of $\sigma_{x}$ in (19)-(20), it is not difficult to show that

$$
(A-K C) \sigma_{x}=-k_{R} B\left[\hat{R} \sigma_{R}\right]_{\times} \hat{R} a_{B} .
$$

Now using the above facts, and in view of $(25)-(27)$, the timescaled dynamics of $\zeta$ are given by

$$
\frac{1}{\gamma} \dot{\zeta}=\left(A-K_{0} C\right) \zeta+\frac{1}{\gamma^{3}} B g\left(t, \tilde{R}, \tilde{b}_{\omega}\right),
$$

where we have defined $g\left(t, \tilde{R}, \tilde{b}_{\omega}\right)_{\tilde{R}}:=(I-\tilde{R})^{\top} \dot{a}_{I}+$ $\tilde{R}^{\top}\left[a_{I}(t)\right]_{\times} R(t) \tilde{b}_{\omega}$. The term $g\left(t, \tilde{R}, \tilde{b}_{\omega}\right)$ is a priori bounded by some constant $c_{g}>0$ in view of Assumptions 23 and the property of the projection mechanism that guarantees boundedness of the bias estimation error $\tilde{b}_{\omega}$. Consider the following real-valued positive function:

$$
\mathbf{V}(\zeta)=\frac{1}{\gamma} \zeta^{\top} P \zeta
$$

where $P$ is the unique solution for the Lyapunov equation $P\left(A-K_{0} C\right)+\left(A-K_{0} C\right)^{\top} P=-I$ which is positive definite thanks to the fact that $\left(A-K_{0} C\right)$ is Hurwitz. Denote by $\beta_{1}$ and $\beta_{2}$ the smallest and largest eigenvalues of $P$, respectively. The time derivative of $\mathbf{V}(\zeta)$ along the trajectories of 40 satisfies

$$
\begin{aligned}
\dot{\mathbf{V}}(\zeta) & =-\|\zeta\|^{2}+\frac{2}{\gamma^{3}} \zeta^{\top} P B g\left(t, \tilde{R}, \tilde{b}_{\omega}\right), \\
& \leq-\frac{1}{2}\|\zeta\|^{2}+\|\zeta\|\left(\frac{2 \beta_{2} c_{g}}{\gamma^{3}}-\frac{1}{2}\|\zeta\|\right), \\
& \leq-\frac{1}{2}\|\zeta\|^{2}, \quad \forall\|\zeta\| \geq \frac{4 \beta_{2} c_{g}}{\gamma^{3}} \\
& \leq-\frac{\gamma}{2 \beta_{2}} \mathbf{V}(\zeta), \quad \forall\|\zeta\| \geq \frac{4 \beta_{2} c_{g}}{\gamma^{3}}
\end{aligned}
$$

Consider the set $\Omega\left(c_{\zeta}\right):=\left\{\zeta: \mathbf{V}(\zeta) \leq \gamma^{-5} c_{\zeta}^{2} \beta_{1}\right\}$ for some $c_{\zeta}>0$. Let us pick $\gamma \geq 4 \beta_{1}^{-\frac{1}{2}} \beta_{2}^{\frac{3}{2}} c_{g} c_{\zeta}^{-1}$. Then, it can be shown that when $\zeta$ is outside the set $\Omega\left(c_{\zeta}\right)$ one has $\|\zeta\|>4 \beta_{2} c_{g} \gamma^{-3}$ and therefore, by (45), the function $V(\zeta)$ is exponentially decreasing outside $\Omega\left(c_{\zeta}\right)$. In this case, $\zeta$ must enter the set $\Omega\left(c_{\zeta}\right)$ before the following time:

$$
\bar{T}^{*}=\frac{2 \beta_{2}}{\gamma} \ln \left(\frac{\gamma \mathbf{V}(\zeta(0))}{\beta_{1} c_{\zeta}^{2}}\right),
$$

which can be tuned arbitrary small by increasing the value of $\gamma$. On the other hand, when $\zeta \in \Omega$ one has $\|\zeta\|^{2} \leq$ $\gamma \beta_{1}^{-1} \mathbf{V}(\zeta) \leq\left(c_{\zeta} \gamma^{-2}\right)^{2}$. The following result immediately follows:

$$
\begin{aligned}
\forall c_{\zeta}, T>0, \forall \zeta(0), \exists \gamma_{1} \geq 1 \text { s.th. } \gamma & \geq \gamma_{1} \Rightarrow \\
\|\zeta(t)\| & \leq \gamma^{-2} c_{\zeta}, \forall t \geq T .
\end{aligned}
$$


This result shows that the gain $\gamma$ can be tuned to guarantee that the error variable $\zeta$ converges arbitrary fast to an arbitrary small ball. Now, we show that the gains can be tuned to guarantee forward invariance of the set $\mho(\epsilon)$. Let $\epsilon \in\left(\frac{1}{2}, 1\right)$ and let the initial attitude estimation error be such that $\tilde{R}(0) \in \mho(\epsilon)$. The time derivative of $|\tilde{R}|^{2}=\operatorname{tr}(I-\tilde{R}) / 4$, in view of 26 and making use of [24, Lemma 2], satisfies

$$
\begin{aligned}
\frac{d}{d t}|\tilde{R}|^{2} & =-\frac{1}{4} \operatorname{tr}(\dot{R}) \\
& =-\frac{1}{4} \operatorname{tr}\left(\mathbf{P}_{\mathfrak{s o}(3)}(\tilde{R})\left[-\hat{R}\left(\tilde{b}_{\omega}+k_{R} \sigma_{R}\right)\right]_{\times}\right) \\
& =-\frac{1}{2} \psi(\tilde{R})^{\top} \hat{R}\left(\tilde{b}_{\omega}+k_{R} \sigma_{R}\right) \\
& \leq\left\|\tilde{b}_{\omega}\right\|+k_{R}\left\|\sigma_{R}\right\| \\
& \leq c_{b}+k_{R}\left(\rho_{1}\left\|m_{I}\right\|^{2}+\rho_{2} c_{2} \hat{c}_{2}\right):=c_{R} .
\end{aligned}
$$

In view of the above inequality on the velocity of $|\tilde{R}|^{2}$, it can be deduced that the minimum time necessary for the attitude estimation error $\tilde{R}$ to leave the set $\mho(\epsilon)$ is greater than

$$
t_{R}:=\frac{\epsilon^{2}-|\tilde{R}(0)|^{2}}{c_{R}} \text {. }
$$

Since we have knowledge about the minimum time necessary for the attitude estimation error to leave the set $\mho(\epsilon)$, it is possible to prevent such a scenario by imposing some (high gain) conditions on the gains $\gamma$ and $k_{R}$ as shown next. Pick $0<T \leq t_{R}$ and $c_{\zeta}=\min \left(\bar{c}_{\zeta} / k_{R}, \hat{c}_{2}-\sqrt{8} c_{2}\right)$ for some arbitrary $\bar{c}_{\zeta}>0$. By (47) there exists $\gamma^{*} \geq 1$ such that if one chooses $\gamma \geq \gamma^{*}$ then $\|\zeta(t)\| \leq \gamma^{-2} c_{\zeta}$ for all $t \geq T$. On the other hand, in view of (28), we have

$$
K_{v}(y-C \hat{x})=-B^{\top} L_{\gamma} \zeta+(I-\tilde{R})^{\top} a_{I} .
$$

Therefore, for all $t \geq T$, one has

$$
\left\|K_{v}(y-C \hat{x})\right\| \leq \gamma^{2}\|\zeta\|+\sqrt{8} c_{2} \leq c_{\zeta}+\sqrt{8} c_{2} \leq \hat{c}_{2} .
$$

Consequently, for all $t \geq T$, the saturation function $\operatorname{sat}_{\hat{c}_{2}}(\cdot)$ in 21] can be removed. Therefore, using [25, Proposition 3], the innovation term $\sigma_{R}$ in 21 can be written as follows:

$$
\begin{aligned}
\sigma_{R} & =\rho_{1}\left(m_{B} \times \hat{R}^{\top} m_{I}\right)+\rho_{2}\left(a_{B} \times \hat{R}^{\top}\left(a_{I}-B^{\top} L_{\gamma} \zeta\right)\right) \\
& =2 \hat{R}^{\top} \psi(M \tilde{R})-\rho_{2}\left(a_{B} \times \hat{R}^{\top} B^{\top} L_{\gamma} \zeta\right),
\end{aligned}
$$

with $M:=\rho_{1} m_{I} m_{I}^{\top}+\rho_{2} a_{I} a_{I}^{\top}$ which is positive semidefinite with an rank of 2 (by Assumption 1). Using the above expression of $\sigma_{R}$, the time derivative of $|\tilde{R}|^{2}$ satisfies

$$
\begin{aligned}
\frac{d}{d t}|\tilde{R}|^{2}= & -k_{R} \psi(\tilde{R})^{\top} \psi(M \tilde{R})-\frac{1}{2} \psi(\tilde{R})^{\top}\left(\hat{R} \tilde{b}_{\omega}-\right. \\
& \left.k_{R} \rho_{2}\left(\hat{R} a_{B}\right) \times B^{\top} L_{\gamma} \zeta\right) \\
\leq & -4 k_{R} \lambda_{\min }^{\mathbf{E}(M)}|\tilde{R}|^{2}\left(1-|\tilde{R}|^{2}\right)+|\tilde{R}|\left\|\tilde{b}_{\omega}\right\|+ \\
& \gamma k_{R} \rho_{2} c_{2}|\tilde{R}|\|\zeta\| \\
\leq & -4 k_{R} \lambda_{\min }^{\mathbf{E}(M)}|\tilde{R}(t)|^{2}\left(1-|\tilde{R}(t)|^{2}\right)+c_{b}+\rho_{2} c_{2} \bar{c}_{\zeta}
\end{aligned}
$$

for all $t \geq T$, where inequalities from [24, Lemma 2] have been used with $\mathbf{E}(M):=\frac{1}{2}\left(\operatorname{tr}(M)-M^{\top}\right)$ for any $M$. Note that the matrix $\mathbf{E}(M)$ is positive definite in view of
Assumption 1. Now assume that $|\tilde{R}(t)|=\epsilon$ and $k_{R}>$ $\left(c_{b}+\rho_{2} c_{2} \bar{c}_{\zeta}\right) /\left(4 \lambda_{\min }^{\mathbf{E}(M)} \epsilon^{2}\left(1-\epsilon^{2}\right)\right)$ then one has

$$
\frac{d}{d t}|\tilde{R}(t)|^{2} \leq-4 k_{R} \lambda_{\min }^{\bar{A}} \epsilon^{2}\left(1-\epsilon^{2}\right)+c_{b}+\rho_{2} c_{2} \bar{c}_{\zeta}<0,
$$

for all $t \geq T$. This implies that $|\tilde{R}(t)|$ is strictly decreasing whenever $|\tilde{R}(t)|=\epsilon$. It follows from the continuity of the solution that $\tilde{R}(t)$ will never leave the ball $\mho(\epsilon)$ for all $t \geq T$. Recall also that $|\tilde{R}(t)| \leq \epsilon$ for all $t \leq T$ (since $T \leq t_{R}$ ). This implies that the set $\mho(\epsilon)$ is forward invariant. Now, let us show the exponential convergence after $t \geq T$. Consider the following Lyapunov function candidate:

$$
\begin{aligned}
\mathbf{W}\left(\zeta, \tilde{R}, \hat{R}, \tilde{b}_{\omega}\right)=|\tilde{R}|^{2}+\frac{\mu k_{R}}{2 k_{b}} \tilde{b}_{\omega}^{\top} \tilde{b}_{\omega}+ & \\
& \mu \tilde{b}_{\omega}^{\top} \hat{R}^{\top} \psi(\tilde{R})+\gamma^{5} \mathbf{V}(\zeta),
\end{aligned}
$$

where $\mu$ is some positive constant scalar and $\mathbf{V}(\zeta)$ is defined in (41). Let $\varsigma:=\left[|\tilde{R}|,\left\|\tilde{b}_{\omega}\right\|,\|\zeta\|\right]^{\top}$ and following a similar procedure to [18, (A.11)-(A.14)], we can show that there exist positive definite matrices $P_{1}, P_{2}, P_{3}$ such that the $\varsigma^{\top} P_{1} \varsigma \leq$ $\mathbf{W} \leq \varsigma^{\top} P_{2} \varsigma$ and $\dot{\mathbf{W}} \leq-\varsigma^{\top} P_{3} \varsigma$ provided that the gains satisfy

$$
\begin{aligned}
& \mu<\lambda_{\min }^{\mathbf{E}(M)}\left(1-\epsilon^{2}\right) / \alpha_{2}, \\
& k_{R}>\max \left\{2 \mu k_{b}, \frac{2 \alpha_{1} \mu^{2}+\left(1+2 c_{\omega} \mu\right)^{2}}{2 \mu \lambda_{\min }^{\mathbf{E}(M)}\left(1-\epsilon^{2}\right)}\right\}, \\
& \gamma>\max \left\{\frac{4\left(\beta_{2}\right)^{2} c_{2}^{2}}{\mu}, \frac{k_{R} \rho_{2} c_{2}+4 \sqrt{2} \beta_{2} c_{3}+\mu\left(\alpha_{3}+k_{R} \alpha_{4}\right)^{2}}{4 k_{R} \lambda_{\min }^{\mathbf{E}(M)}\left(1-\epsilon^{2}\right)}\right\},
\end{aligned}
$$

where $\left.\alpha_{1}=8 c_{b}^{2}+4 k_{b} \lambda_{\max }^{\mathbf{E}(M)}, \alpha_{2}=8 \lambda_{\max }^{\mathbf{E}(M)} c_{b}(\sqrt{2}+4)\right)$, $\alpha_{3}=2 k_{b} \rho_{2} c_{2}$ and $\alpha_{4}=2 \rho_{2} c_{b} c_{2}(\sqrt{2}+4)$. Consequently, the error variable $\varsigma$ converges exponentially to zero after $t \geq T$.

\section{REFERENCES}

[1] D. Titterton, J. L. Weston, and J. Weston, Strapdown inertial navigation technology. IET, 2004, vol. 17.

[2] O. J. Woodman, "An introduction to inertial navigation," Technical Report, University of Cambridge Computer, Laboratory, no. UCAMCL-TR-696, pp. 1-37, 2007.

[3] B. Vik and T. I. Fossen, "A nonlinear observer for gps and ins integration," in Proceedings of the 40th IEEE Conference on Decision and Control (Cat. No. 01CH37228), vol. 3, 2001, pp. 2956-2961.

[4] J. Farrell, Aided navigation: GPS with high rate sensors. McGraw-Hill, Inc., 2008.

[5] H. F. Grip, T. I. Fossen, T. A. Johansen, and A. Saberi, "Nonlinear Observer for GNSS-Aided Inertial Navigation with Quaternion-Based Attitude Estimation," in American Control Conference, 2013, pp. 272279.

[6] T. A. Johansen and T. I. Fossen, "Nonlinear observer for inertial navigation aided by pseudo-range and range-rate measurements," in European Control Conference (ECC), 2015, pp. 1673-1680.

[7] T. A. Johansen, J. M. Hansen, and T. I. Fossen, "Nonlinear Observer for Tightly Integrated Inertial Navigation Aided by Pseudo-Range Measurements," Journal of Dynamic Systems, Measurement, and Control, vol. 139, no. 1, pp. 1-10, 2017.

[8] T. H. Bryne, J. M. Hansen, R. H. Rogne, N. Sokolova, T. I. Fossen, and T. A. Johansen, "Nonlinear Observers for Integrated Implementation Aspects," IEEE Control Systems Magazine, vol. 37, no. 3, pp. 59-86, 2017.

[9] K. Gryte, J. M. Hansen, T. Johansen, and T. I. Fossen, "Robust navigation of UAV using inertial sensors aided by UWB and RTK GPS," in AIAA Guidance, Navigation, and Control Conference, 2017, pp. 1-16.

[10] M. Hamer and R. D'Andrea, "Self-calibrating ultra-wideband network supporting multi-robot localization," IEEE Access, vol. 6, pp. 22292 22304,2018 
[11] A. M. Sabatini, "Quaternion-based extended kalman filter for determining orientation by inertial and magnetic sensing," IEEE transactions on Biomedical Engineering, vol. 53, no. 7, pp. 1346-1356, 2006.

[12] J. L. Crassidis, "Sigma-point Kalman filtering for integrated GPS and inertial navigation," IEEE Transactions on Aerospace and Electronic Systems, vol. 42, no. 2, pp. 750-756, 2006.

[13] M. Whittaker and J. L. Crassidis, "Inertial Navigation Employing Common Frame Error Representations," AIAA Guidance, Navigation, and Control Conference, pp. 1-24, 2017.

[14] J. M. Hansen, T. A. Johansen, N. Sokolova, and T. I. Fossen, "Nonlinear observer for tightly coupled integrated inertial navigation aided by rtkgnss measurements," IEEE Transactions on Control Systems Technology, vol. 27, no. 3, pp. 1084-1099, 2018.

[15] S. Berkane and A. Tayebi, "Position, velocity, attitude and gyro-bias estimation from imu and position information," in 18th European Control Conference (ECC), 2019, pp. 4028-4033.

[16] M. Wang and A. Tayebi, "Hybrid nonlinear observers for inertial navigation using landmark measurements," IEEE Transactions on Automatic Control, vol. 65, no. 12, pp. 5173-5188, 2020.

[17] _ - "Nonlinear state estimation for inertial navigation systems with intermittent measurements," Automatica, vol. 122, pp. 1-11, 2020.

[18] S. Berkane, A. Tayebi, and S. de Marco, "A nonlinear navigation observer using imu and generic position information," Automatica, vol. 127, p. 109513, 2021.

[19] M. Krstic, I. Kanellakopoulos, and P. V. Kokotovic, Nonlinear and adaptive control design. Wiley New York, 1995.

[20] R. Mahony, T. Hamel, and J.-m. Pflimlin, "Nonlinear Complementary Filters on the Special Orthogonal Group," IEEE Transactions on Automatic Control, vol. 53, no. 5, pp. 1203-1218, 2008.

[21] T.-T. Lu and S.-H. Shiou, "Inverses of $2 \times 2$ block matrices," Computers \& Mathematics with Applications, vol. 43, no. 1-2, pp. 119-129, 2002.

[22] F. Esfandiari and H. K. Khalil, "Output feedback stabilization of fully linearizable systems," International Journal of control, vol. 56, no. 5, pp. 1007-1037, 1992.

[23] A. Saberi and P. Sannuti, "Observer design for loop transfer recovery and for uncertain dynamical systems," IEEE Transactions on Automatic Control, vol. 35, no. 8, pp. 878-897, 1990.

[24] S. Berkane, A. Abdessameud, and A. Tayebi, "Hybrid Attitude and Gyro-bias Observer Design on SO(3)," IEEE Transactions on Automatic Control, vol. 62, no. 11, pp. 6044-6050, 2017.

[25] S. Berkane and A. Tayebi, "Construction of Synergistic Potential Functions on $\mathrm{SO}(3)$ with Application to Velocity-Free Hybrid Attitude Stabilization," IEEE Transactions on Automatic Control, vol. 62, no. 1, pp. 495-501, 2017. 